\title{
The 21st International Symposium on Spin Physics (Spin2014)
}

Monday 20 October 2014 - Friday 24 October 2014

\author{
Peking University, Beijing, China.
}

Programme 


\section{Monday 20 October 2014}

Opening and Plenary Session I (Chair: Haiyan Gao)- (08:15-10:00)

\begin{tabular}{|c|c|c|}
\hline time & title & presenter \\
\hline $08: 15$ & Welcome Remarks & $\begin{array}{l}\text { XIE, Xincheng (Dean of School } \\
\text { of Physics, Peking Univ.) }\end{array}$ \\
\hline $08: 30$ & Spin Structure of the Nucleon & JI, Xiangdong (SJTU/UMD) \\
\hline 09:00 & Parity-Violating Electron Scattering and the Weak Charge of the Proton & MACK, Dave (JLab) \\
\hline 09:30 & Parity Violations in Hadronic Systems & SNOW, William (Indiana Univ.) \\
\hline
\end{tabular}

\section{Symposium Photo and Coffee Break - (10:00-10:30)}

Plenary Session II (Chair: Thomas Roser) - (10:30-12:00)

\begin{tabular}{|c|c|c|}
\hline time & title & presenter \\
\hline 10:30 & Beam Polarization at the ILC: Physics Case and Realisation & VAUTH, Annika (DESY) \\
\hline 11:00 & Production of High Nuclear Spin Alignment of Radioactive Ion Beams & UENO, Hideki (RIKEN) \\
\hline $11: 30$ & Physics with Polarized Targets in Storage Ring & $\begin{array}{l}\text { TOPORKOV, Dmitriy (BINP } \\
\text { and NSU) }\end{array}$ \\
\hline
\end{tabular}

Lunch Break - (12:00-13:30)

Parallel-I: S1 (Chair: Fan Wang) - (13:30-15:15)

\begin{tabular}{|l|l|}
$\begin{array}{l}\text { time title } \\
\text { 13:30 Recent Developments in Nucleon Spin Decomposition }\end{array}$ & presenter \\
\hline 14:00 Nucleon Tomography: Wigner Distributions & $\begin{array}{l}\text { HATTA, Yoshitaka } \\
\text { (Japan/Yukawa Institute) }\end{array}$ \\
\hline 14:30 Extracting PDFs by Global Fit of Lattice QCD Calculations & $\begin{array}{l}\text { PASQUINI, Barbara (University } \\
\text { of Pavia) }\end{array}$ \\
\hline 14:55 Perturbative Matching of the Quasi-PDFs in Continuum Space and Lattice Space & MA, Yan-Qing (BNL) \\
\hline
\end{tabular}

Parallel-I: S5 (Chair: Xiaomei Li)- (13:30-15:15)

time title

13:30 Transverse Spin Asymmetries in the CNI Region of Elastic Proton-Proton Scattering at $\$$ lsqrt $\{$ s $\$=200 \mathrm{GeV}$

14:00 Measurement of the Analyzing Power in Proton-Proton Elastic Scattering at Small Angles

14:25 Study of the \$leta Meson Production with Polarized Proton Beam

14:50 Initial Research of $n p$ Scattering with Polarized Deuterium Target at ANKE/COSY

Parallel-I: S8 (Chair: Jens Erler) - (13:30-15:15) presenter

SVIRIDA, Dmitry (ITEP)

MACHARASHVILI, Giorgi

(Joint Institute for Nuclear Research)

ZIELINSKI, Marcin (Jagiellonian University)

GOU, Boxing (Institute of Modern Physics, Chinese Academy of Sciences) 
13:30 Recent Results and Progress on Leptonic and Storage Ring EDM Searches

14:00 Measurement of Muon g-2/EDM with Ultra-cold Muon at J-PARC

14:20 The New Muon g-2 Experiment at Fermilab

14:40 High-precision Microwave Spectroscopy of Muonium for Determination of Muonic Magnetic Moment

Parallel-I: S9 (Chair: Andreas Lehrach)- (13:30-15:15)

time title

13:30 Polarimetry for Stored Polarized Hadron Beams

13:55 High Precision Electron Beam Polarimetry

14:20 RHIC Proton Polarimetry Current Status and Future Plans

14:38 Absolute and Relative Polarimeters for the SPACHARM Experiment.

14:56 The Deuteron Beam Polarimetry at Nuclotron-NICA

\section{Coffee Break - (15:15-15:30)}

Parallel-ll: S1 (Chair: Jianwei Qiu) - (15:30-17:30)

time title

15:30 Hadron Structure from Drell-Yan Processes: An Overview

16:00 Overview of TMD Evolution

16:30 Gluon TMDs and Quarkonium Production in Unpolarised and Polarised Proton-Proton Collisions

16:50 Some New Opportunities for Spin Physics at Small $x$

Parallel-Il: S5 (Chair: Yajun Mao) - (15:30-17:30)

time title

15:30 Spin Physics with PHENIX Experiment's MPC-EX Calorimeter Upgrade.

16:00 Azimuthal Asymmetries of Drell-Yan Process in pA Collisions

16:25 High Twist Effects in $\$ \mathrm{e}^{\wedge}+\mathrm{e}^{\wedge}-\$$ Annihilation at High Energies

Parallel-ll: S8 (Chair: Krishna Kumar) - (15:30-17:30) presenter

KAWALL, David (University of Massachusetts Amherst)

MIBE, Tsutomu (IPNS, KEK)

LI, Liang (Shanghai Jiao Tong University (CN))

TORII, Hiroyuki A. (University of Tokyo)

presenter

STEPHENSON, Edward (Indiana University)

DUTTA, Dipangkar (Mississippi State University)

MAKDISI, Yousef (BNL)

SEMENOV, Pavel (IHEP)

LADYGIN, Vladimir (Joint Institute for Nuclear Research (JINR))

presenter

GROSSE-PERDEKAMP,

Matthias (Univ. Illinois at

Urbana-Champaign (US))

BOER, Daniel (University of Groningen)

LANSBERG, Jean-Philippe (IPN Orsay, Paris Sud U. / IN2P3-CNRS)

ZHOU, Jian (Regensburg University)

JIANG, Xiaodong (Los Alamos National Laboratory)

GAO, Jian-Hua (Shandong University at Weihai)

WEI, Shu-yi (School of Physics, Shandong University) 
15:30 Parity Violation in Deep Inelastic Scattering with the SoLID Spectrometer at JLab

16:00 Searches for Physics beyond the Standard Model at the LHC
SOUDER, Paul (Syracuse University)

ELLINGHAUS, Frank

(Johannes-Gutenberg-Universitaet Mainz (DE))

16:30 Towards a Precision Measurement of the Muon Pair Asymmetry in e+e- Annihilation at Belle and Belle II

16:50 Test of Time-Reversal Invariance at COSY (TRIC)

FERBER, Torben (DESY)

EVERSHEIM, Dieter

(Helmholtz Institut für Strahlenund Kernphysik, University Bonn, Germany)

17:10 Test of Time-reversal Symmetry in the Proton - Deuteron Scattering UZIKOV, Yuriy (Joint Institue for Nuclear Researches)

Parallel-II: S9 (Chair: Mei Bai) - (15:30-17:30)

time title presenter

15:30 Effect of Overlapping Intrinsic Spin Resonances on e-lens lattices from FY13 Polarized Proton Run

15:55 High energy Polarized Electrons

RANJBAR, Vahid (BNL)

SHATUNOV, Yury (Budker institute of nuclear physics)

16:20 Polarization Preservation and Control in a Figure-8 Ring MOROZOV, Vasiliy (Jefferson Lab, Newport News, VA, USA)

16:45 Beam Polarization Aspects of eRHIC PTITSYN, Vadim (Brookhaven National Laboratory)

17:05 Study of the Polarization Deterioration During Physics Stores in RHIC Polarized Proton Runs DUAN, Zhe (institute of high energy physics, Chinese Academy of Sciences)

Parallel-II: S12 (Chair: Xincheng Xie) - (15:30-17:30)

time title presenter

15:30 Quantum Computation and Quantum Metrology Based on Solid Spin System DU, Jiangfeng (USTC)

16:00 Quantum Information Processing Based on NV Centers PAN, Xin-Yu (loP, CAS)

16:30 Quantum Computing and Entanglement Purification on Electron Spins DENG, Fu-Guo (Beijing Normal Univ.)

17:00 Quantum Simulation with Nuclear Spins
LONG, Gui-Lu (Tsinghua Univ.) 


\section{Tuesday 21 October 2014}

Plenary Session III (Chair: Oleg Teryaev) - (08:00-10:00)

time title

presenter

08:00 Lattice QCD for Spin Physics

LIU, Keh-Fei (Univ. of

Kentucky)

08:30 Nucleon Electromagnetic Form Factors

CATES, Gordon (Univ. of Virginia)

09:00 Latest Results from the COMPASS Experiment STOLARSKI, Marcin (LIP)

09:30 Results from RHIC Spin Program

RENEE, Fatemi (Univ. of Kentucky)

\section{Coffee Break - (10:00-10:15)}

Plenary Session IV (Chair: Franco Bradamante) - (10:15-12:00)

time title

presenter

10:15 Highlights from HERMES

ROSTOMYAN, Armine (DESY)

10:45 Summary of PSTP2013

POELKER, Matthew (JLab)

11:00 Summary of D-SPIN2013

EFREMOV, Anatoly V. (JINR)

11:15 Summary of SPIN-Praha-2013

FINGER, Michael (Prague)

11:30 Medical Imaging with Highly Spin Polarized Molecules

WARREN, Warren (Duke Univ.)

\section{Lunch Break - (12:00-13:30)}

Parallel-IIl: S2 (Chair: Jian-Ping Chen) - (13:30-15:15)

time title

presenter

13:30 Theoretical Status of Helicity Parton Densities

NOCERA, Emanuele Roberto

(Università degli Studi di Milano \& INFN Milano, Italy)

14:00 New Results on the Longitudinal Spin Structure of the Nucleon from CLAS at Jefferson Lab

BOSTED, Peter (College of William and Mary)

14:30 COMPASS Results on $\mathrm{g} 1$ and NLO QCD Fits

KUNNE, Fabienne

(CEA/IRFU,Centre d'etude de Saclay Gif-sur-Yvette (FR))

Parallel-III: S4 (Chair: Nicole D'Hose) - (13:30-15:15)

time title

presenter

13:30 Nucleon Form Factors

BRISCOE, Bill (George Washington Univ.)

14:00 Overview of the Proton Radius Problem

CARLSON, Carl (William and Mary)

14:30 New Precision Measurement for Proton Zemach Radius with Laser Spectroscopy of Muonic Hydrogen

MA, Yue (RIKEN) 
Parallel-IIl: S5 (Chair: Nikolai Kochelev) - (13:30-15:15)

time title

presenter

13:30 Role of Spin in $\mathrm{NN} \rightarrow \mathrm{NN} \pi$

BARU, Vadim (Ruhr University Bochum)

14:00 Spin Physics at NICA

NAGAYTSEV, Alexander (JINR Dubna on behalf SPD team)

Parallel-III: S8 (Chair: Wei-Tou Ni) - (13:30-15:15)

time title

presenter

13:30 Spin-gravity Interactions and Equivalence Principle

TERYAEV, Oleg (JINR)

14:18 New Limit on Lorentz-Invariance- and CPT-Violating Neutron Spin Interactions Using a Free-Spin-Precession $\$^{\wedge} 3 \$ \mathrm{He}-\$^{\wedge}\{129\} \$$ Xe Comagnetometer

ALLMENDINGER, Fabian (Physikalisches Institut, Uni Heidelberg, Germany)

14:37 Limits for Spin-Dependent Short-Range Interaction of Axion-like Particles

TULLNEY, Kathlynne (University Mainz, Germany)

14:56 New Spin and Velocity Dependent Force Searching by Using Polarized Helium Gas

FU, Changbo (Shanghai Jiaotong University)

Parallel-III: S12 (Chair: Gui Lu Long) - (13:30-15:15)

time title

presenter

13:30 Planar Quantum Squeezing and Optimized Interferometric Phase Measurement HE, Qiongyi (Peking Univ.)

14:00 Quantum Information Processing Based on Quantum-dots in Optical Double-sided Microcavities

14:25 Demonstrating a Quantum Algorithm Using Nuclear Magnetic Resonance WANG, Tie-jun (Beijing U Post and Communications)

LIU, Yang (North China Electric Power University)

\section{Coffee Break - (15:15-15:30)}

Parallel-IV: S2 (Chair: Renee Fatemi) - (15:30-17:30)

time title

15:30 Proton Quark Helicity Structure via W-boson Production in pp Collision at Isqrt(s) $=500 \mathrm{GeV}$ @ PHENIX

16:00 Proton Spin Content in Lattice QCD from a Large Momentum Effective Field Theory

16:15 Measurement of Longitudinal Spin Asymmetries for Weak Boson Production in Polarized Proton-Proton Collisions at STAR

16:30 Symmetry Breaking and Determination of Strange Quark Distribution of the Nucleon

16:45 Accessing Polarized Sea Flavor Asymmetry through Semi-Inclusive DIS at JLab-12GeV and the Future EIC presenter

GIORDANO, Francesca (UIUC)

ZHAO, Yong (University of Maryland)

ZHANG, Jinlong (Shandong University/Brookhaven Nationa Laboratory)

CAO, Fu-Guang (Massey University)

JIANG, Xiaodong (Los Alamos National Laboratory)

Parallel-IV: S4 (Chair: Barbara Pasquini) - (15:30-17:30)

time title

15:30 Nucleon Form Factors: An Incisive Window into Quark-Gluon Dynamics presenter

CLOET, Ian (Argonne National Laboratory) 
16:00 Theory and Phenomenology of GPDs

KUMERICKI, Kresimir

(University of Zagreb)

16:30 Progress in Double Distributions and Generalized Parton Distributions Modeling

MEZRAG, Cedric (IRFU/SPhN)

16:50 Generalized Parton Distributions for the Nucleon in the Soft-wall Model of AdS/QCD

SHARMA, Neetika (Indian institute of Science Education and Research Mohali)

Parallel-IV: S6 (Chair: Reinhard Beck) - (15:30-17:30)

time title

presenter

15:30 Double Polarisation Experiments in Meson Photoproduction and the Impact on the Nucleon Excitation Spectrum

HARTMANN, Jan (HISKP Univerity of Bonn)

16:00 Double Polarisation Experiments in Meson Photoproduction at JLab PASYUK, Eugene (Jefferson Lab)

16:30 Spin Density Matrix Elements in Exclusive Production of Omega Mesons at HERMES MARUKYAN, Hrachya (DESY)

Parallel-IV: S9 (Chair: Edward Stephenson)- (15:30-17:30)

time title presenter

15:30 Storage Ring Based EDM Search

LEHRACH, Andreas

(Forschungszentrum Juelich)

15:55 Systematic Calculation of Spin Resonance Strengths to High Order

BARBER, Desmond

(Deutsches Elektronen

Synchrotron (DESY),

Germany.)

16:15 Studies of Systematic Limitations in the EDM Searches at Storage Rings

SALEEV, Artem

(Forschungszentrum Juelich)

16:30 A Novel RF-ExB Spin Manipulator at COSY (Jülich, Germany)

MEY, Sebastian

(Forschungszentrum Juelich)

16:45 Machine Development for Spin

LENISA, Paolo (University of Ferrara and INFN)

Parallel-IV: S12 (Chair: Warren Warren)- (15:30-17:30)

time title

presenter

15:30 Dipolar and Quadrupolar Signatures of Topological Band Structures BOUCHARD, Louis (UCLA)

16:00 Polarized Fusion: Can Polarization Help to Increase the Energy Output of Fusion Reactors?

16:25 Polarized Fusion and its Implications: The Potential for Direct in situ Measurements of Fuel Polarization Survival in a Tokamak Plasma

ENGELS, Ralf (FZ Jülich)

SANDORFI, Andrew (Jefferson Lab)

16:50 Generation of States with Long-lived Molecular Polarization and Catalytic Generation In-Magnet of Molecular Spin Hyperpolarization

WARREN, Warren (Duke Univ.)

17:10 Recent Trends in Laser-polarized Gases for Medical Imaging and Nuclear Targets

CATES, Gordon (Univ. of Virginia) 


\section{Wednesday 22 October 2014}

Parallel-V: S2 (Bo-Wen Xiao) - (08:15-10:15)

time title

presenter

08:15 Constraints on $\triangle \mathrm{G}$ from COMPASS Data

KUREK, Krzysztof (National

Centre for Nuclear Research $(\mathrm{PL})$ )

08:45 Gluon Polarization in Longitudinally Polarized $\$ p p \$$ Collisions at STAR

CHANG, Zilong (Texas A\&M University)

09:15 Impact of PHENIX Measurements of $\$ A_{-}\{L L\}^{\wedge}\left\{\mid p^{\wedge}{ }^{\wedge} 0\right\} \$$ on Determination of the Gluon Spin in the Proton

MANION, Andrew (Lawrence Berkeley National Laboratory)

09:30 Recent Results of $\$ A \_\{L L\}^{\wedge}\left\{\mid p^{\wedge} 0\right\} \$$ Measurements at $\$$ Isqrt $\{s\}=510 \$ \mathrm{GeV}$ at Mid-rapidity by YOON, Inseok (Seoul National PHENIX Experiment and Resulting Constraint on the Gluon Spin Contribution to the Proton Spin University)

09:45 \$J/psi\$ Longitudinal Double Spin Asymmetry Measurement at Forward Rapidity in \$p+p\$ Collisions at $\$$ lsqrt $\{\mathrm{s}\}=510 \$ \mathrm{GeV}$

YU, Haiwang (PKU, NMSU, LANL)

10:00 Double Helicity Asymmetries of Forward Neutral Pions from $\$ \mid s q r t\{s\}=510 \$ \mathrm{GeV} \$ p p \$$

DILKS, Christopher Collisions at STAR

Parallel-V: S3 (Chair: Xiang-Song Chen) - (08:15-10:15)

time title presenter

08:15 Transverse Momentum and Spin Dependent Distribution Functions MULDERS, Piet J. (VU/Nikhef)

08:45 Recent Key Measurements for Accessing the Transverse Spin and Momentum Structure of the Nucleon

MARTIN, Anna (Trieste University and INFN (IT))

09:15 Transverse Spin Azimuthal Asymmetries in SIDIS at COMPASS

PARSAMYAN, Bakur (University of Turin and INFN (IT))

09:30 Transversity Experiment (E06-010) at Jlab ZHAO, Yuxiang (USTC)

09:45 Transverse Single-spin Asymmetries of Pion Production in Semi-inclusive DIS at Subleading MAO, Weijuan (Southeast Twist University)

10:00 Momentum Structure of the Nucleon and Hadron Production in Unpolarised SIDIS at COMPASS

MAKKE, Nour (Universita e INFN (IT))

Parallel-V: S6 (Chair: Xiaodong Jiang) - (08:15-10:15)

time title

presenter

08:15 Spin Physics with Photons - Technical Highlights and Spin-offs

THOMAS, Andreas (University Mainz)

08:45 Spin Degrees of Freedom in Compton Scattering

MISKIMEN, Rory (University of Massachusetts)

09:15 Spin Observables in Pion Photoproduction and Nucleon Compton Scattering from the Chiral Lagrangian and Dispersion Relations.

GASPARYAN, Ashot (Ruhr University of Bochum)

09:35 Transverse and Longitudinal Lambda Polarization in Lepton Scattering by Unpolarized Nucleons at HERMES

KARYAN, Gevorg (DESY)

Parallel-V: S9 (Chair: Vasiliy Morozov)- (08:15-10:15) 
08:15 NICA Facility in Polarized Proton and Deuteron Mode

KOVALENKO, Alexander (Joint Institute for Nuclear Research)

08:40 Multiple Horizontal Tune Jumps To Overcome Horizontal Depolarizing Resonances

HUANG, Haixin (Brookhaven National Lab)

08:58 A New Formalism for Classifying Spin Motions Using Tools Distilled from the Theory of

BARBER, Desmond

Principal Bundles

(Deutsches Elektronen

Synchrotron (DESY),

Germany)

09:16 Calculation of Spin Resonances with Strong Betatron Coupling

PTITSYN, Vadim (Brookhaven National Laboratory)

09:34 Estimation of Systematic Errors for Deuteron Electric Dipole Moment (EDM) Search at a

CHEKMENEV, Stanislav

Storage Ring

(Rheinisch-Westfälische

Technische Hochschule (RWTH))

09:52 Electron Polarization In The MEIC Collider Ring At JLab

LIN, Fanglei (Thomas

Jefferson National Accelerator

Facility)

\section{Coffee Break - (10:15-10:30)}

Parallel-Vl: S2 (Chair: Peter Bosted) - (10:30-12:00)

time title presenter

10:30 Study of g2 Spin Structure Function at Jefferson Lab

$\mathrm{CHOI}$, Seonho (Seoul National University)

11:00 A Measurement of $\$ g \_2^{\wedge} p \$$ at Low $\$ Q^{\wedge} 2 \$$

CUMMINGS, Melissa (The College of William and Mary)

11:15 Quark to the \$ILambda \$ and \$Ibar ILambda\$ Spin Transfers in the Current Fragmentation Region

DU, Xiaozhen (Peking University)

11:30 COMPASS Results on Hadron Multiplicities and Fragmentation Functions

KUNNE, Fabienne

(CEA/IRFU,Centre d'etude de Saclay Gif-sur-Yvette (FR))

11:45 Multiplicities of Charged Pions and Kaons in Deep-inelastic Scattering by Protons and Deuterons at HERMES and the Strange-quark Distribution in the Nucleon

KARYAN, Gevorg (DESY)

Parallel-Vl: S3 (Chair: Matthias Grosse-Perdekamp) - (10:30-12:00)

time title

presenter

10:30 Fragmentation Function Measurements at Belle

GIORDANO, Francesca (UIUC)

10:45 New COMPASS Results on Transverse Spin Asymmetries in Hadron Pair Production in DIS

SBRIZZAI, Giulio (Trieste Univ and INFN)

11:00 QCD Evolution Effects for the Collins Azimuthal Asymmetries in e+e- and SIDIS

SUN, Peng (LBNL)

11:15 Measurement of Double Collins Asymmetries at BESIII

GUAN, Yinghui (IHEP)

11:30 Phenomenological Extraction of Transversity from COMPASS SIDIS and Belle e+e- Data

BRADAMANTE, Franco

(Universita e INFN (IT))

Parallel-VI: S6 (Chair: Reinhard Beck) - (10:30-12:00) 
10:30 Complete Experiments in Meson Photoproduction

WUNDERLICH, Yannick (University of Bonn)

11:00 Unraveling Excitations of the Nucleon - Meson Photo-production from Polarized Neutrons in $\mathrm{HD}$ at CLAS

SANDORFI, Andrew (Jefferson Lab)

Parallel-VI: S7 (Chair: Zhigang Xiao) - (10:30-12:00)

time title

presenter

10:30 Novel Spin Modes in Exotic Nucleus

MENG, Jie (Peking University)

11:00 N3LO Chiral Predictions for Spin Observables in Nucleon-Deuteron Elastic Scattering and the SKIBINSKI, Roman Deuteron Breakup at Low Energies. (Jagiellonian University)

11:20 Study of Three Nucleon Force Effects via Few-Nucleon Scattering WADA, Yasunori (Tohoku university)

11:40 Polarization Effects in Deuteron-induced Reactions OU, Li (Guangxi Normal University)

Parallel-Vl: S10 (Chair: Matt Poelker) - (10:30-12:00)

time title presenter

10:30 Review of Polarized lon Sources ZELENSKI, Anatoli (BNL)

11:00 Design of Transversal Phase Space Meter for Atomic Hydrogen Beam Source BELOV, Alexander (INR)

11:20 Polarized $\mathrm{He} 3$ lon Source for RHIC and eRHIC MAXWELL, James (MIT)

11:40 Status of the New Source of Polarized lons for the JINR Accelerator Complex FIMUSHKIN, Victor (Joint Institute for Nuclear Research)

Parallel-VI: S11 (Chair: Jianwei Qiu) - (10:30-12:00)

time title

presenter

10:30 Systematic Study of Spin Effects at SPASCHARM Experiment MOCHALOV, Vasily (IHEP, Protvino)

10:50 New $p+p$ and $p+A$ Physics Opportunities with the Forward sPHENIX Upgrade at the Relativisitc Heavy Ion Collider

LIU, Ming Xiong (Los Alamos National Laboratory)

11:10 MPD and BM@N Detectors at NICA. Prospects for the Polarization Effects Measurements. PESHEKHONOV, Dmitry (Joint Institute for Nuclear Research)

\section{Lunch Break - (12:00-13:30)}

Excursion - (13:30-17:30)

Public Lecture - (19:00-20:00)

time title

presenter

19:00 Quantum Anomalous Hall Effect and Information Technology XUE, Qi-Kun (Tsinghua Univ.) 


\section{Thursday 23 October 2014}

Plenary Session V (Chair: Anna Martin)- (08:15-10:15)

\begin{tabular}{l|l|l|}
$\begin{array}{l}\text { time } \\
\text { title }\end{array}$ & presenter \\
\hline 08:15 & Three-dimensional Imaging of the Nucleon: TMD (Theory/Phenomenology) & $\begin{array}{l}\text { LIANG, Zuo-Tang (Shandong } \\
\text { Univ.) }\end{array}$ \\
\hline 08:45 Spin Physics with 12-GeV CEBAF & ENT, Rolf (JLab) \\
\hline 09:15 Future Spin Physics at RHIC & BOYLE, Kieran (BNL) \\
\hline 09:45 EPJ A Lecture: Spin Physics at an Electron-lon Collider & $\begin{array}{l}\text { MEZIANI, Zein-Eddine (Temple } \\
\text { Univ.) }\end{array}$ \\
\hline
\end{tabular}

\section{Coffee Break - (10:15-10:30)}

Plenary Session VI (Chair: Hans Stroeher) - (10:30-12:00)

time title

presenter

10:30 Spin Physics at J-PARC

KUMANO, Shunzo (KEK)

11:00 Spin Physics at COSY - Recent Results and Future Plans

KACHARAVA, Andro (Forschungszentrum Juelich)

11:30 Latest Results on Few-body Physics AHMED, Mohammad (NCCU and TUNL)

\section{Lunch Break - (12:00-14:00)}

Memorial Session in Honor of M. Borghini (Chair: Alan Krisch) - (14:00-16:00)

time title

presenter

14:00 Introduction

$\mathrm{KRISCH}$, Alan D. (University of Michigan)

14:15 The Dawn of High Energy Spin Physics MASAIKE, Akira (Kyoto Univ.)

14:55 Today's Polarized Targets in Borghini's Footsteps

MEYER, Werner Peter (Ruhr-Universitaet Bochum (DE))

15:25 Borghini's Contributions to Today's Polarized Targets CRABB, Donald G. (University of Virginia)

15:50 Borghini as a mentor at CERN

Poster Session (Chair: Xiaomei Li) - (14:00-16:00)

time title

presenter

15:00 Coffee Hour

Banquet - (18:30-21:00) 


\section{Friday 24 October 2014}

Parallel-VIl: S3 (Chair: Han-Xin He)- (08:15-10:15)

time title

08:15 Key Future Measurements of TMDs at JLab and Other Facilities

presenter

ALLADA, Kalyan

(Massachusetts Institute of Technology)

08:45 Transverse Target Single-spin Asymmetry in Inclusive Electroproduction of Charged Pions and Kaons

SCHNELL, Gunar (DESY)

09:00 Azimuthal Asymmetries for eA/eN Semi-inclusive DIS and Its Nuclear Dependence

SONG, Yu-kun (Jinan Univ.)

09:15 Transverse Single Spin Asymmetries of Forward $\$ \mid p_{i} \wedge\{0\} \$$ and Jet-like Events in $\$ \mid s q r t\{s\} \$=$ $500 \mathrm{GeV}$ Polarized Proton Collisions at STAR

PAN, Yuxi (University of California, Los Angeles)

09:30 Measuring Transversity with Di-hadron Correlations in $p \$\{\wedge$ \{luparrow $\}\}+p$ Collisions at $\$$ lsqrt $\{$ s $\$=500 \mathrm{GeV}$

SKOBY, Michael (Indiana University)

09:45 New COMPASS Work on the interplay among $\mathrm{h}+, \mathrm{h}-$, and $2 \mathrm{~h}$ transverse spin asymmetries in BRADAMANTE, Franco (trieste SIDIS

university and infn)

10:00 Azimuthal Single-Spin Asymmetries of Charged Pions in Jets in $\$ \mid s q r t\{s\}=200 \$ \mathrm{GeV}$ $\$ p^{\wedge}\{$ luparrow\}p $\$$ Collisions at STAR

ADKINS, J. Kevin (University of Kentucky)

Parallel-VII: S4 (Chair: Nicole D'Hose) - (08:15-10:15)

time title

presenter

08:15 An Experimental Overview on DVCS Measurements (Past, Present and Future)

FISCHER, Horst

(Albert-Ludwigs-Universitaet

Freiburg (DE))

08:45 DVCS at HERMES

MARUKYAN, Hrachya (DESY)

09:05 The DVCS Physics Program at COMPASS

FERRERO, Andrea

(Commissariat a l'Energie

Atomique et aux Energies

Alternatives)

09:25 Deeply Virtual Meson Production at Jefferson Lab

KUBAROVSKY, Valery

(Jefferson Lab)

09:45 Hard Exclusive Meson Production at COMPASS

TER WOLBEEK, Johannes (Albert-Ludwigs-Universitaet Freiburg (DE))

Parallel-VIl: S7 (Chair: Kimiko Sekiguchi) - (08:15-10:15)

time title

presenter

08:15 Studies of Unstable Nuclei with Spin-Polarized Proton Target

SAKAGUCHI, Satoshi (Kyushu University)

08:45 Polarized Proton Elastic Scattering and Nucleon Density Distributions ZENIHIRO, JuZo (RIKEN)

09:05 Relativistic Studies of Spin-isospin Response in Nuclei

LIANG, Haozhao (RIKEN Nishina Center)

09:25 Recent Progress in Spin-isospin Excitations in Nuclei

HIROYUKI, Sagawa (RIKEN/University of Aizu)

09:45 Spin Polarization of Radioisotope Atoms with Optical Pumping in Superfluid Helium for the Measurement of Nuclear Spins and Electromagnetic Moments

FURUKAWA, Takeshi

(Department of Physics, Tokyo Metropolitan University) 
Parallel-VIl: S10 (Chair: Don Crabb) - (08:15-10:15)

time title

presenter

08:15 Review of Solid Polarized Targets

KEITH, C. (JLab)

08:45 High Deuteron Polarization in Polymer Target Materials

WANG, Li (Donghua University)

09:00 Tensor Polarization Optimization and Measurement for Solid Spin 1 Targets

KELLER, Dustin (University of Virginia)

09:20 A New Polarized Solid Proton Target for Fermilab E1039 Drell-Yan Experiment

LIU, Ming (Los Alamos National Laboratory)

09:35 Preparations for Electron Beam Experiments with Transversely Polarized Solid HD

WEI, Xiangdong (Jefferson Lab)

09:55 The $\mathrm{H}$ and D Polarized Target for Spin-filtering Measurements at COSY

CIULLO, Giuseppe (INFN and Dipartimento di Fisica e Scienze della Terra - 44121 Ferrara Italy)

Parallel-VIl: S11 (Chair: Rolf Ent) - (08:15-10:15)

time title

presenter

$08: 15$ EIC in the US

$\mathrm{BAI}, \mathrm{Mei}(\mathrm{BNL})$

08:45 EIC/HIAF in China

CHEN, Xurong (IMP)

09:15 ePHENIX: An Electron Ion Collider (EIC) Detector Built Around the BaBar Magnet

BAZILEVSKY, Alexander (Brookhaven National Laboratory)

09:35 TMD Studies with A Fixed-Target ExpeRiment Using the LHC Beams (AFTER@LHC) MASSACRIER, Laure Marie (LAL/IPNO)

09:55 TMD Studies and More with SoLID at JLab CHEN, Jian-Ping (JLab)

Parallel-VII: S12 (Chair: Xi Chen) - (08:15-10:15)

time title

presenter

08:15 Spin Superconductor and Electric Dipole Superconductor

XIE, Xincheng (Peking Univ.)

08:50 Aharonov-Casher Effect in $\mathrm{Bi} 2 \mathrm{Se} 3$ Square-Ring Interferometers

LU, Li (Institute of Physics, Chinese Academy of Sciences)

09:25 Real-space Imaging of Dirac-Laudau Orbits in Topological Surface State

FU, Yingshuang (Huazhong Univ. of Science \& Technology)

09:45 Spin-wave Nanograting Coupler YU, Haiming (Beihang Univ.)

Coffee Break - (10:15-10:30)

Parallel-VIII: S3 (Chair: Ming Liu) - (10:30-12:00)

time title

presenter

10:30 Measurement of Boer-Mulders Function via Drell-Yan Process by SeaQuest Experiment at Fermilab

NAKANO, Kenichi (Tokyo Tech)

10:45 Working With Wilson Lines

VAN DER VEKEN, Frederik (University of Antwerp)

11:00 Transverse Single-spin Asymmetry of Heavy-flavor Production in $\$ p+p \$$ Collisions at $\$$ lsqrt $\{$ s $\$=200 \mathrm{GeV}$ 
11:15 Gluon Contribution to the Sivers Effect COMPASS Results on Deuteron Target.

11:30 Transverse Single Spin Asymmetry of $\$ \mid \mathrm{pi}^{\wedge} 0 \$$ and $\$$ leta $\$$ Mesons at RHIC/PHENIX

11:45 Single-spin Asymmetries in SIDIS on the Longitudinally Polarized Nucleon Targets
KUREK, Krzysztof (National Centre for Nuclear Research $(\mathrm{PL}))$

XIAORONG, Wang (New Mexico State University and RBRC)

LU, Zhun (Southeast University)

Parallel-VIII: S4 (Chair: Chuan Liu) - (10:30-12:00)

time title

presenter

10:30 Nucleon Structure from Lattice QCD

ZANOTTI, James (University of Adelaide)

11:00 Parton Distribution Functions and Matching

ZHANG, Jianhui (SJTU)

11:20 Quark Angular Momentum in a Spectator Model

LIU, Tianbo (Peking University)

11:40 Quark Wigner Distributions and Orbital Angular Momentum in Light-front Dressed Quark Model

NAIR, Sreeraj (Indian Institute of Technology, Bombay)

\section{Parallel-VIII: S7 (Chair: Seonho Choi) - (10:30-12:00)}

time title

presenter

10:30 Polarization Observables in Few-Nucleon Scattering

KISTRYN, Stanislaw (Jagiellonian University Krakow)

11:00 Development of Neutron Polarization Measurement System for Studying NN Interaction in Nuclear Medium

YASUDA, Jumpei (Department of Physics, Kyushu University)

11:20 Spin-orbit Splitting of Oxygen Isotopes Studied via (pol p, 2p) Reaction KAWASE, Shoichiro (the University of Tokyo)

11:40 The Spin Studies in Few Body Systems at Nuclotron LADYGIN, Vladimir (LHEP-JINR)

Parallel-VIII: S10 (Chair: Dipangkar Dutta) - (10:30-12:00)

time title

presenter

10:30 High Current Electron Guns for eRHIC

BEN-ZVI, Ilan (Brookhaven National Laboratory)

11:00 DC-SRF Photo-cathode Gun at Peking University

LIU, Kexin (Peking University)

11:25 Overview of Polarized He3 Gas Targets CHEN, Jian-ping (Jefferson Lab)

Parallel-VIII: S11 (Chair: Alexander Nagaytsev) - (10:30-12:00)

time title

presenter

10:30 Opportunities with Polarized Hadron Beams

LORENZON, Wolfgang (Michigan)

11:00 Polarized Drell-Yan at COMPASS-II: Transverse Spin Physics Program

PARSAMYAN, Bakur

(University of Turin and INFN

(IT))

11:20 Cross Section and Asymmetry Measurement of Very Forward Neutral Particle Production at $\mathrm{RHIC}$

GOTO, Yuji (RIKEN) 
11:40 Nucleon Partonic Spin Structure to be explored by the Unpolarized Drell-Yan Program in the COMPASS-II Experiment at CERN

CHANG, Wen-Chen (Academia Sinica (TW))

Parallel-VIII: S12 (Chair: Li Lu) - (10:30-12:00)

time title presenter

10:30 Quantum Anomalous Hall Effect in Magnetically Doped Topological Insulator $\mathrm{HE}, \mathrm{Ke}$ (Tsinghua Univ.)

11:00 U(1) Symmetry Protected Spin Quantum Hall Effect in S = 1 Gutzwiller Wavefunction LIU, Zhengxin (Tsinghua Univ.)

11:20 Observation of the Surface States in Topological Kondo Insulator SmB6 and YbB6 ZHANG, Tong (Tsinghua Univ.)

11:40 Dynamical Generation of Fermion Mass in Weyl Semimetals WANG, Zhong (Tsinghua Univ.)

\section{Lunch Break - (12:00-13:30)}

Plenary Session VII (Chair: Erhard Steffens) - (13:30-15:45)

time title

presenter

13:30 Searches for EDMs

14:00 Low-Energy Tests of Standard Model

FILIPPONE, Brad (Caltech)

4:30 Search for the Role of Spin and Polarization in Gravity

MAAS, Frank (Mainz)

$15: 00$

NI, Wei-Tou (National Tsinghua Univ.)

15:00 Symposium Summary MILNER, Richard (MIT)

15:30 Symposium Closing 


\section{The 21st International Symposium on Spin Physics (Spin2014)}

Monday 20 October 2014 - Friday 24 October 2014

Peking University, Beijing, China.

List of Participants 
$1111 \mathrm{~m} / \mathrm{m} \mid \mathrm{n}$

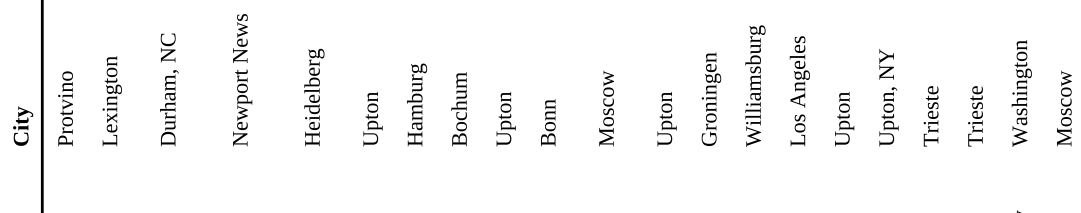

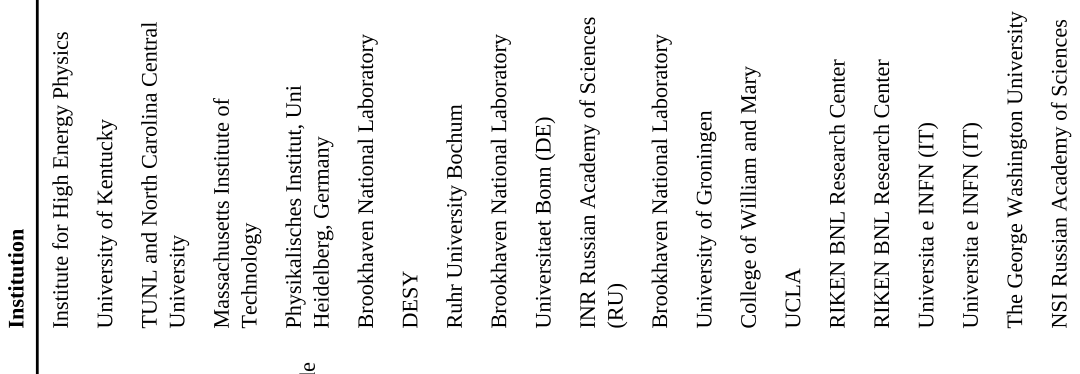

|| $\mid$

III In In In 


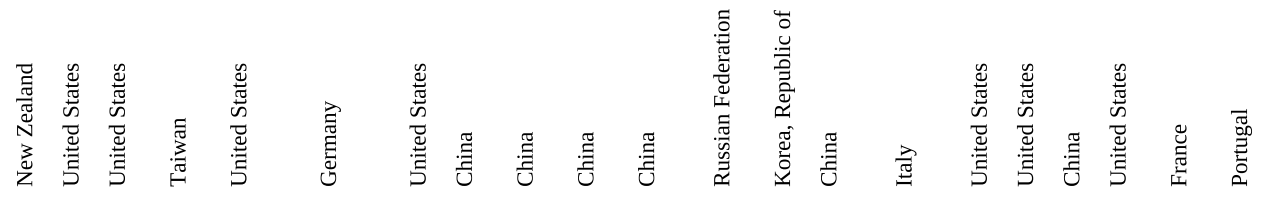

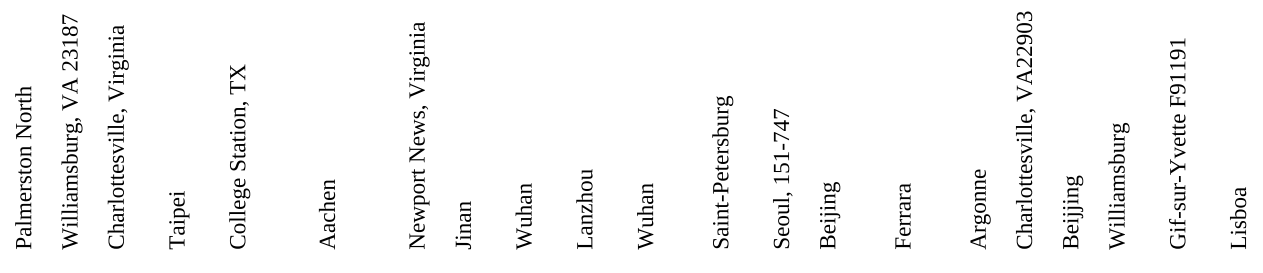

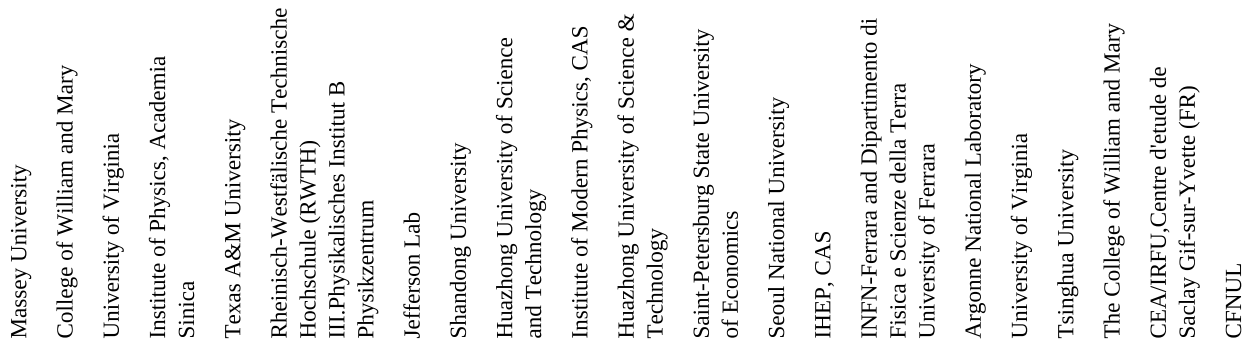

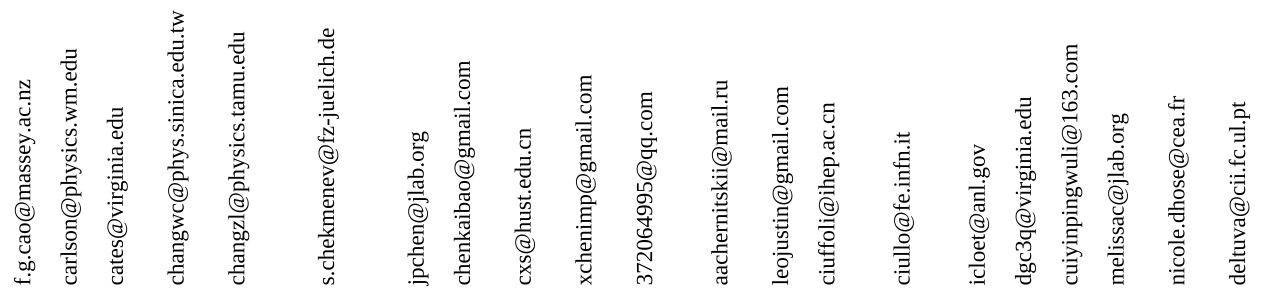

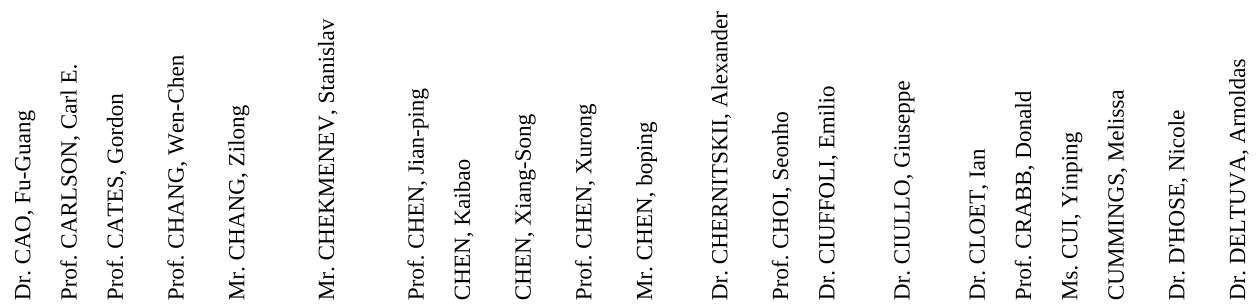




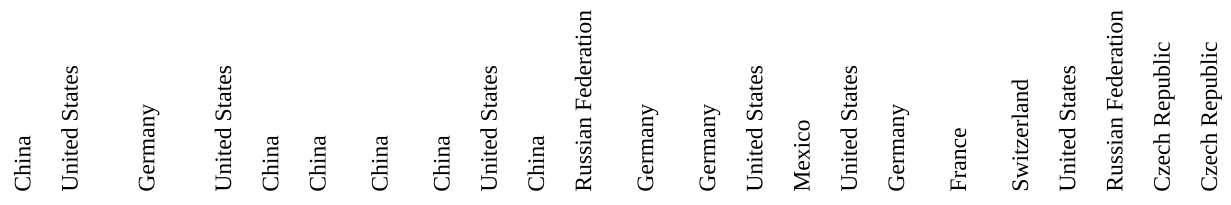

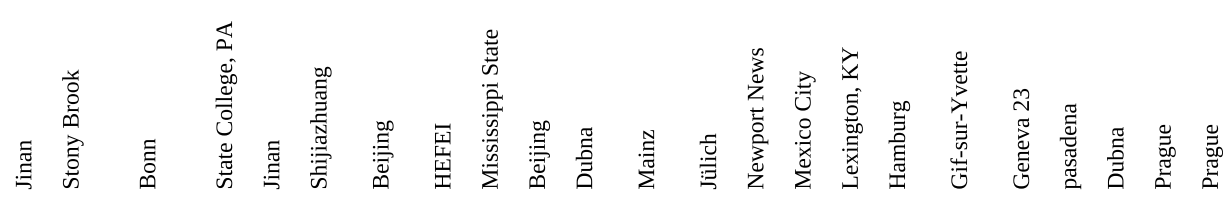

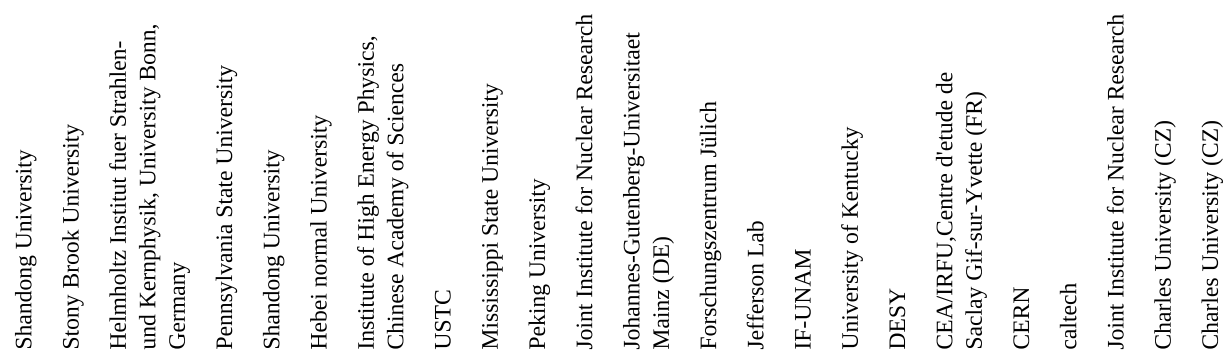

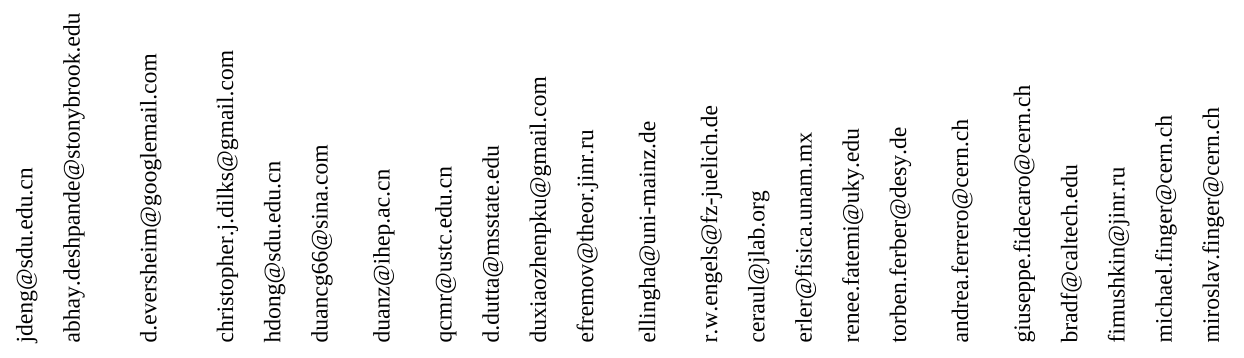

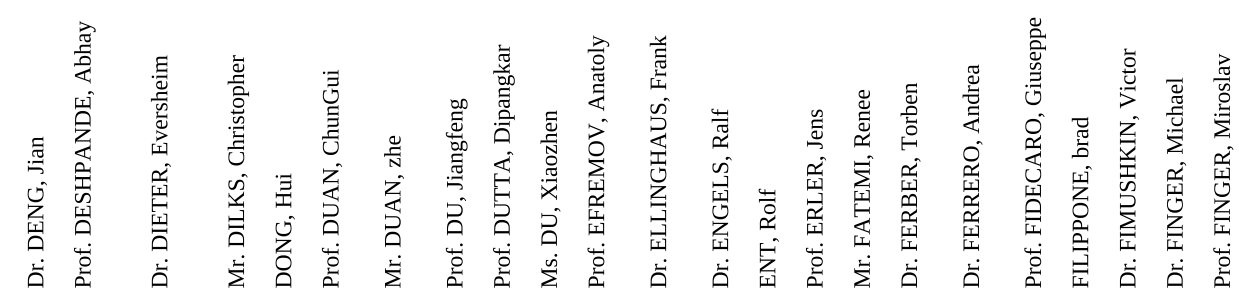




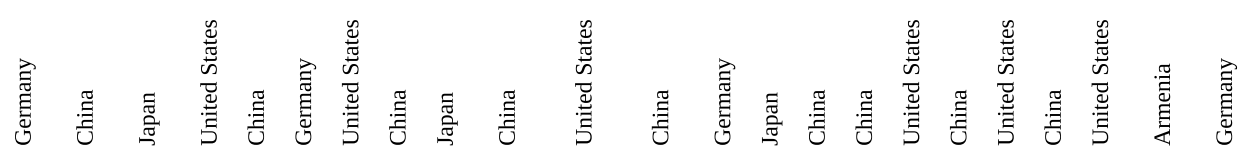<smiles>[AlH2][AlH2]</smiles>

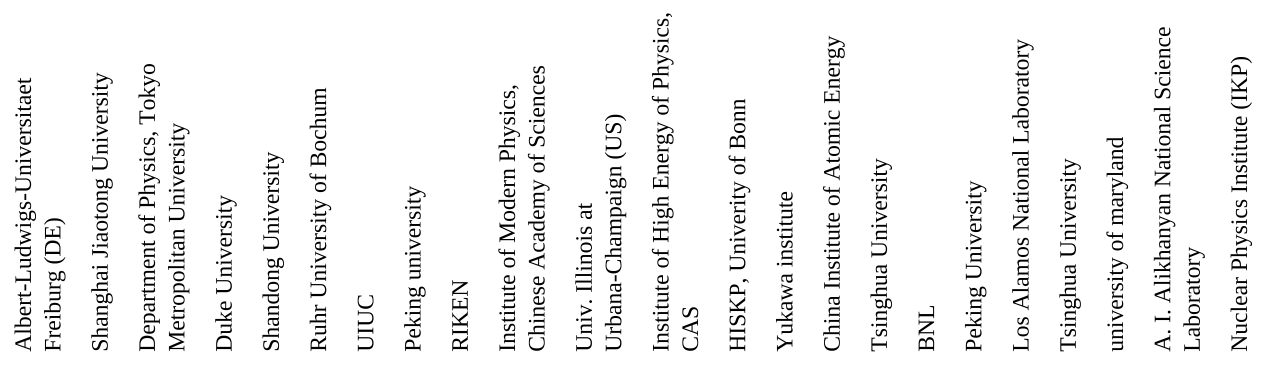

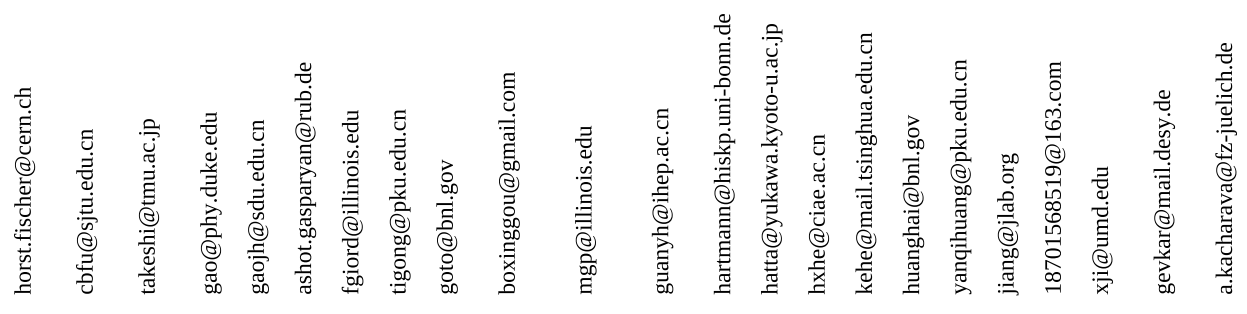

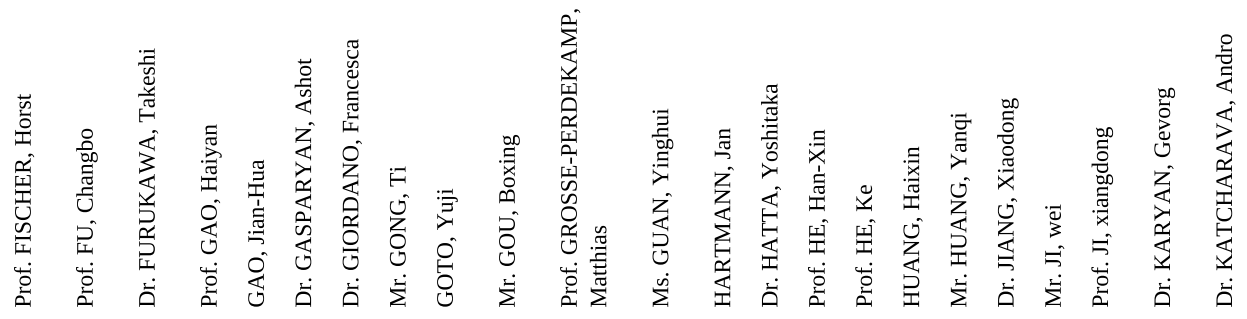




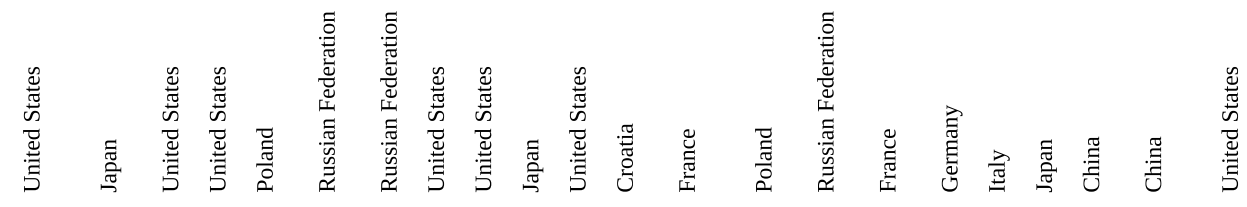<smiles>[134IH]</smiles>

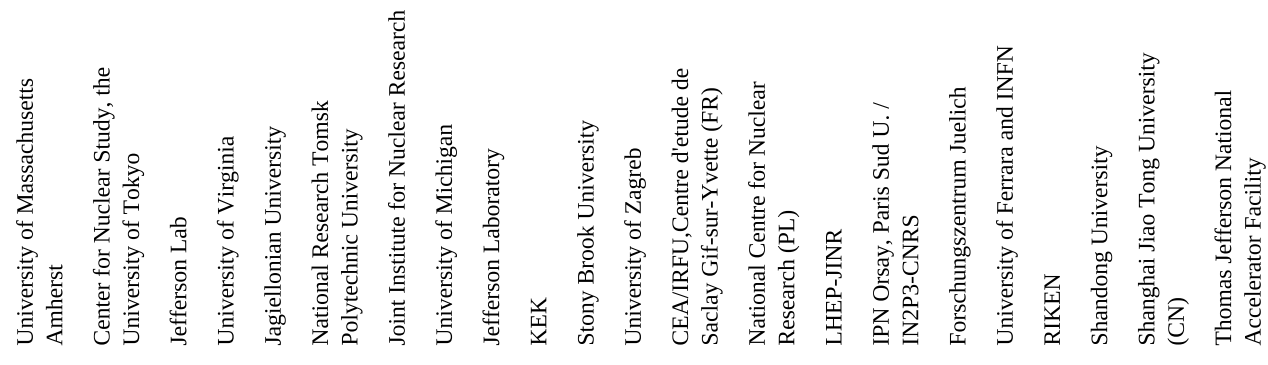

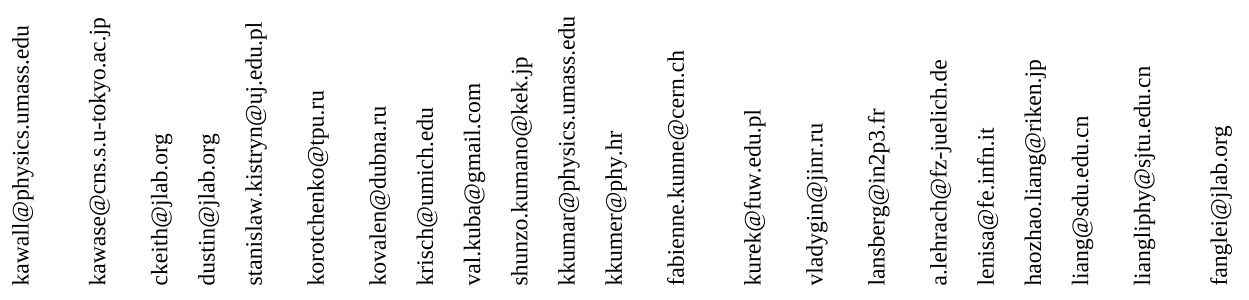

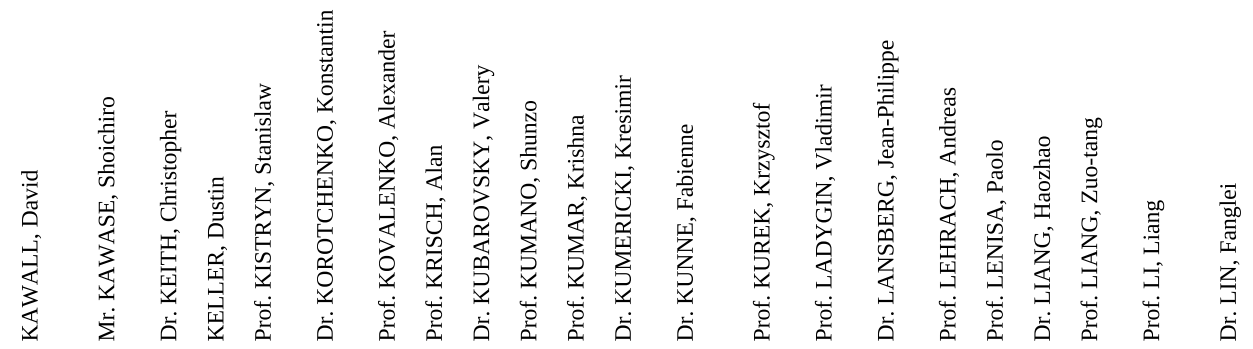




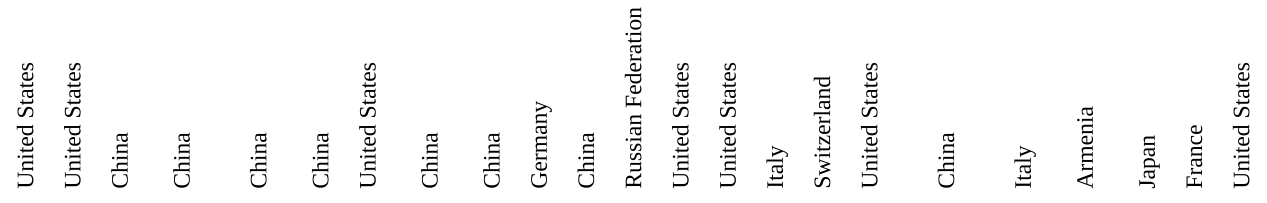

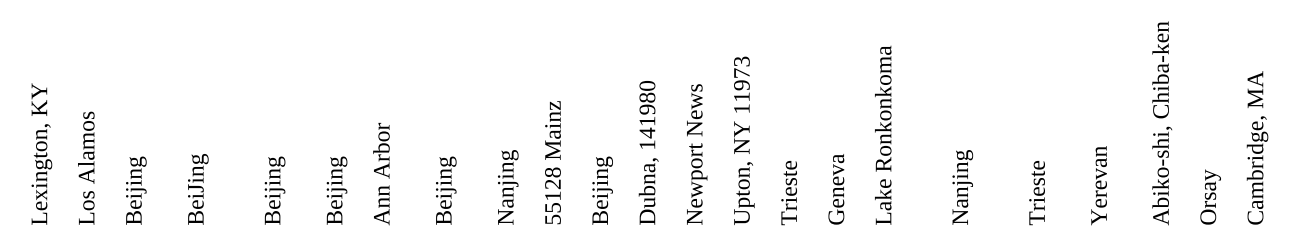

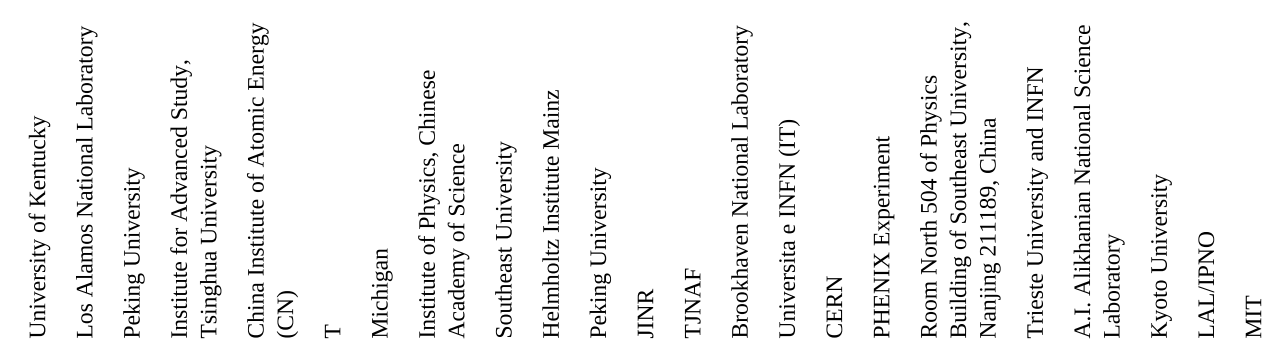

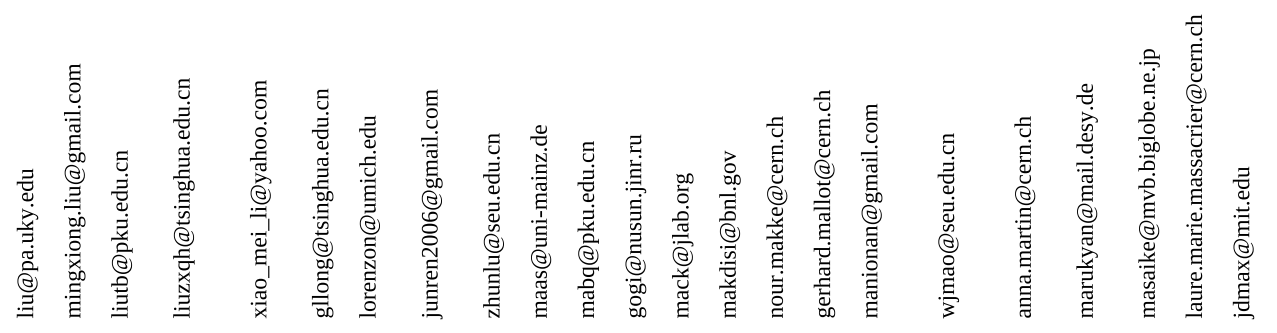

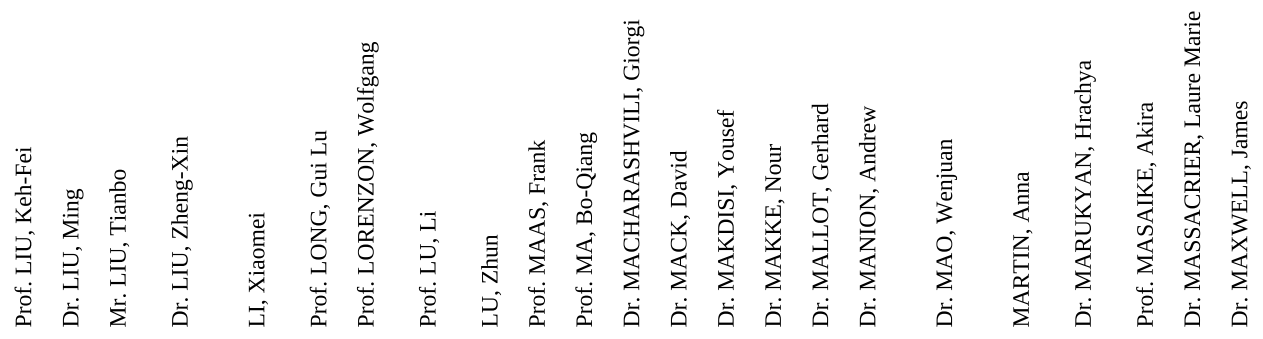




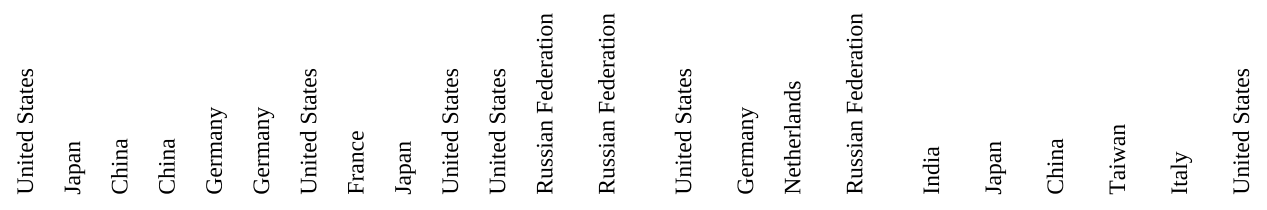<smiles>[AlH2]</smiles>

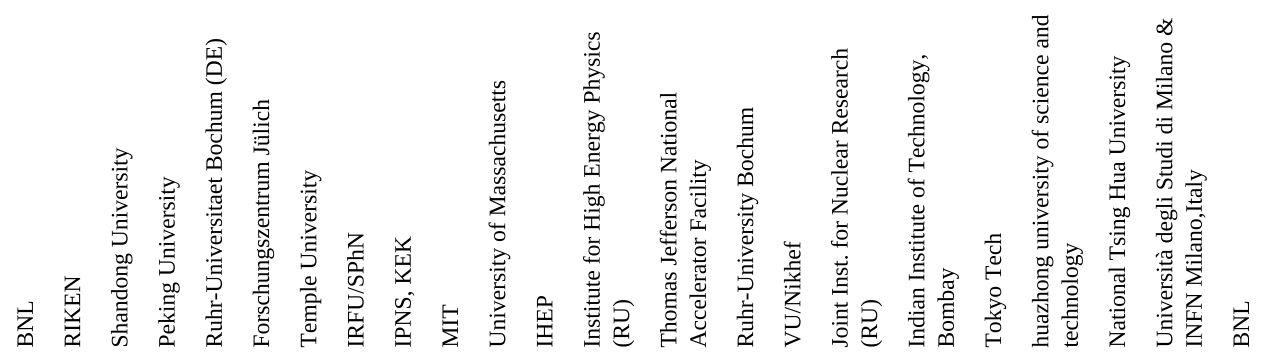

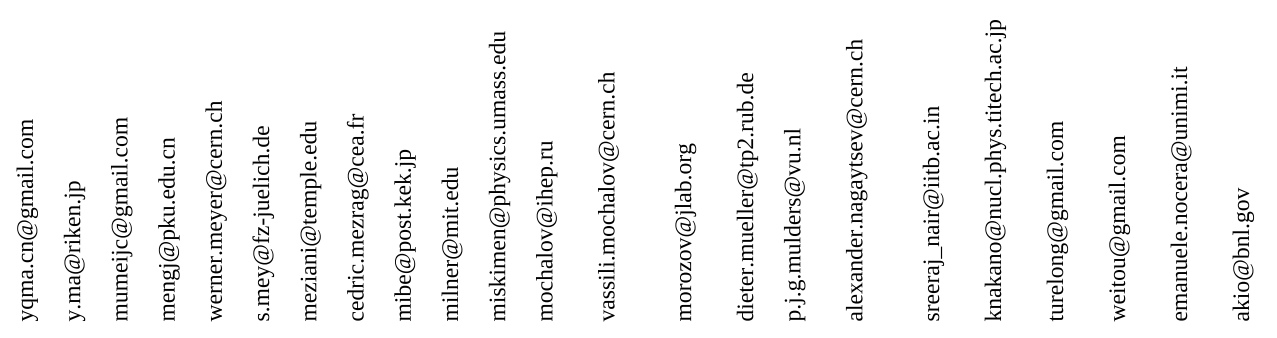

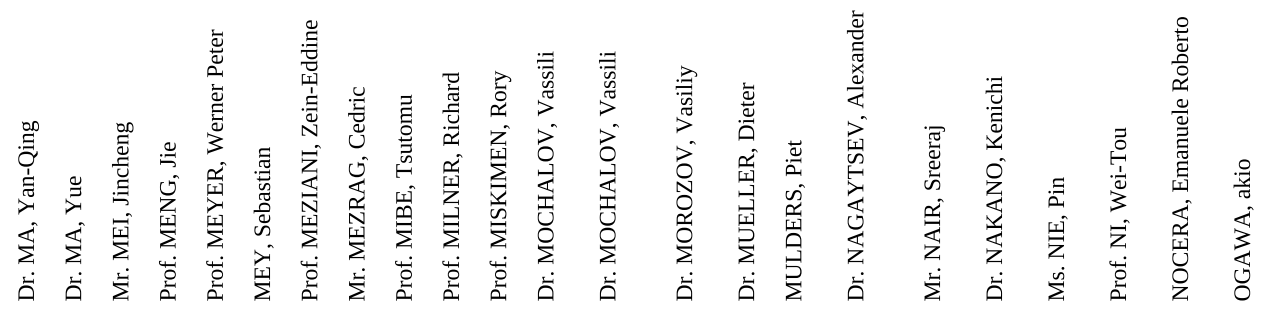




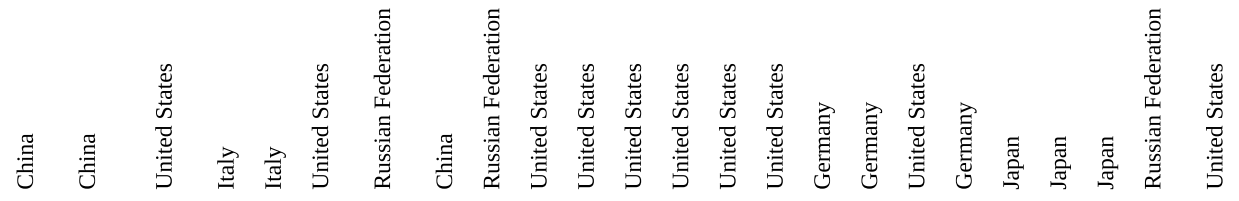

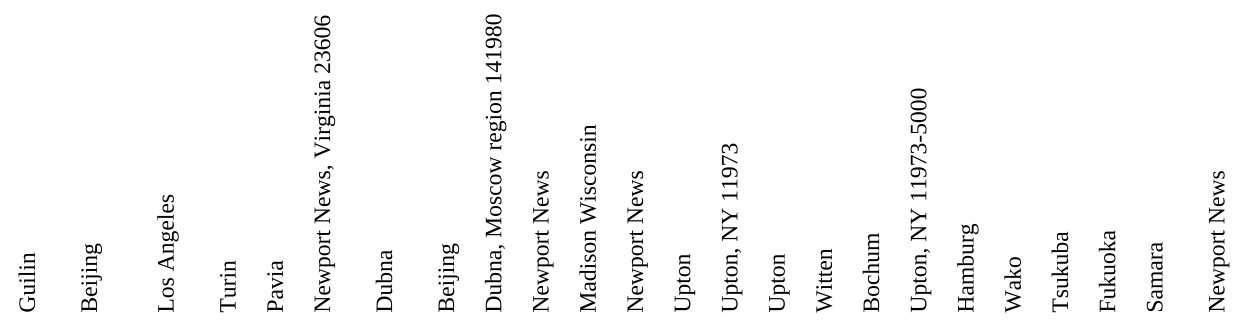

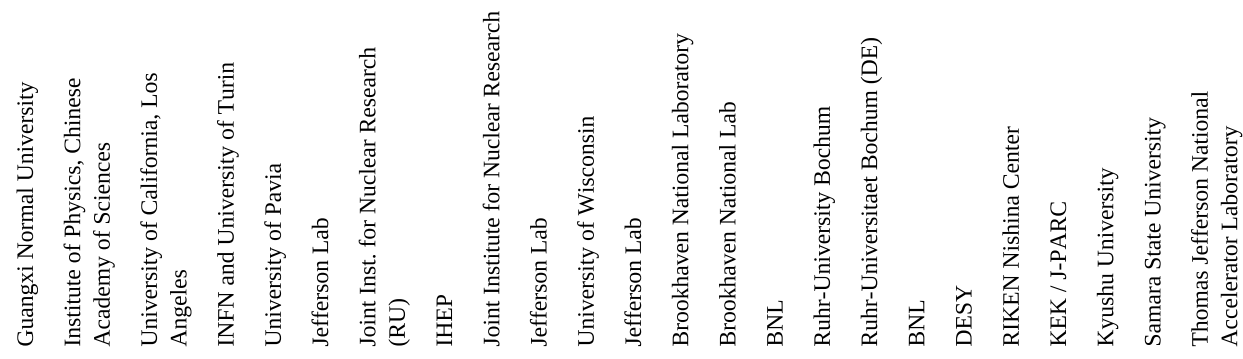

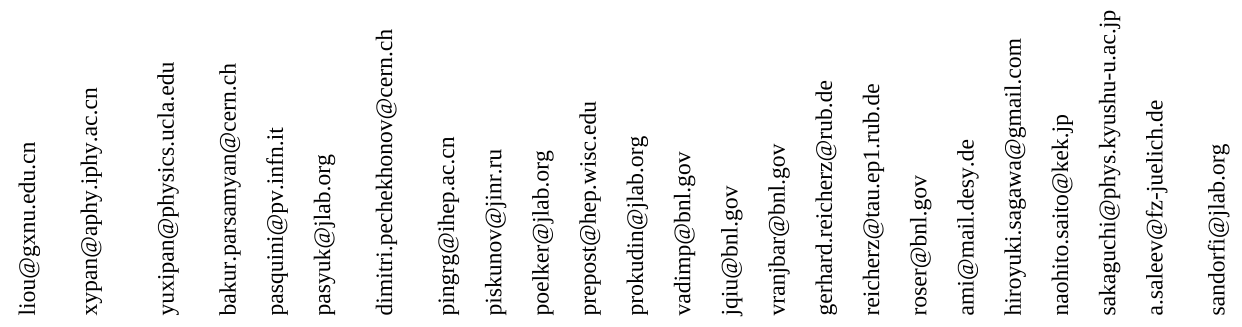

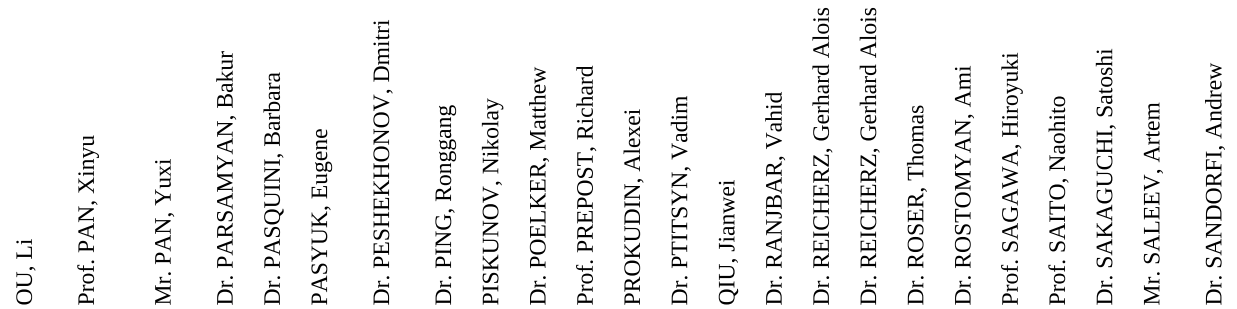




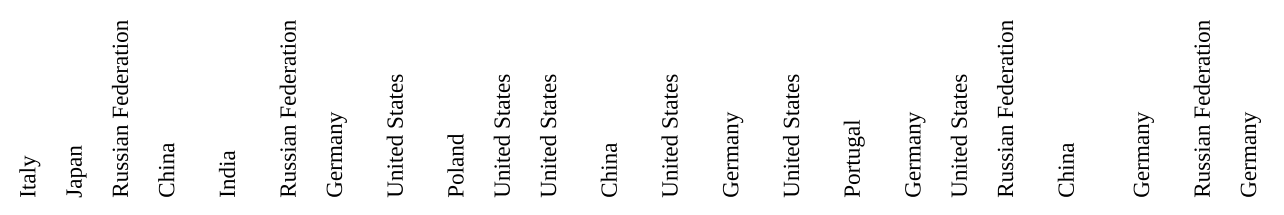<smiles>[AlH2][AlH2]</smiles>

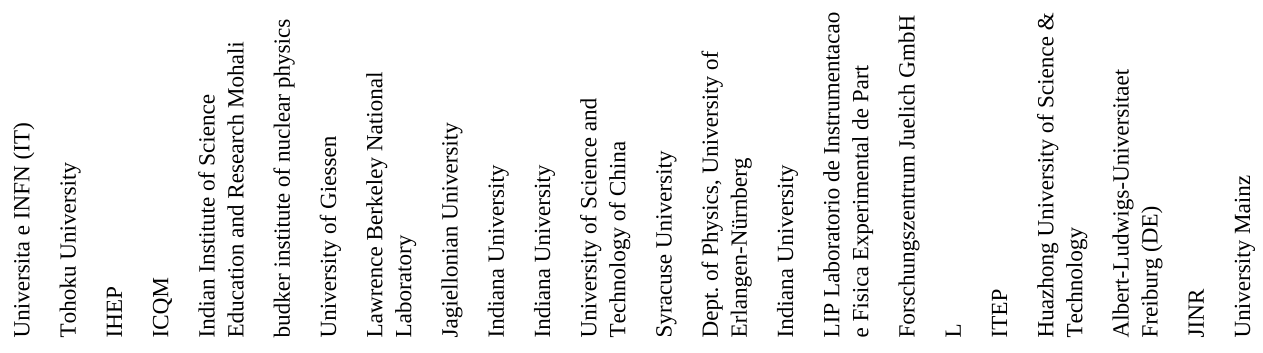

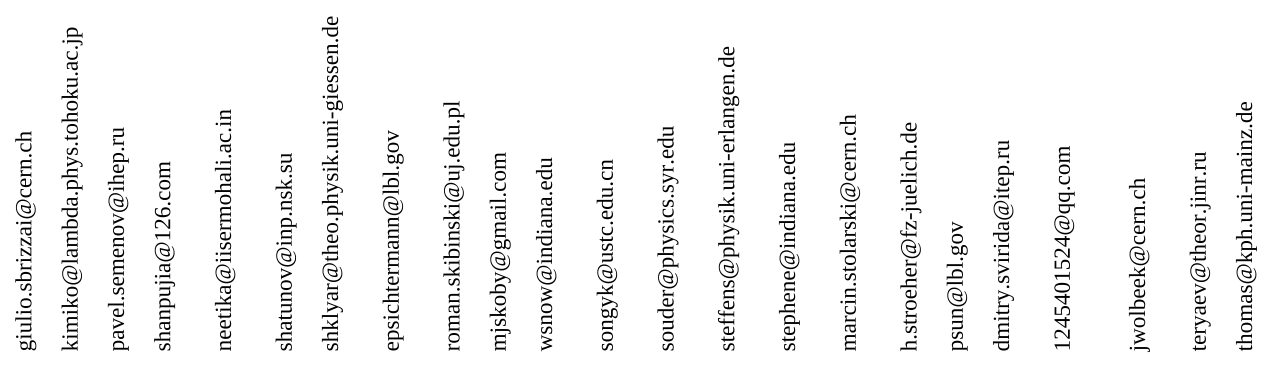

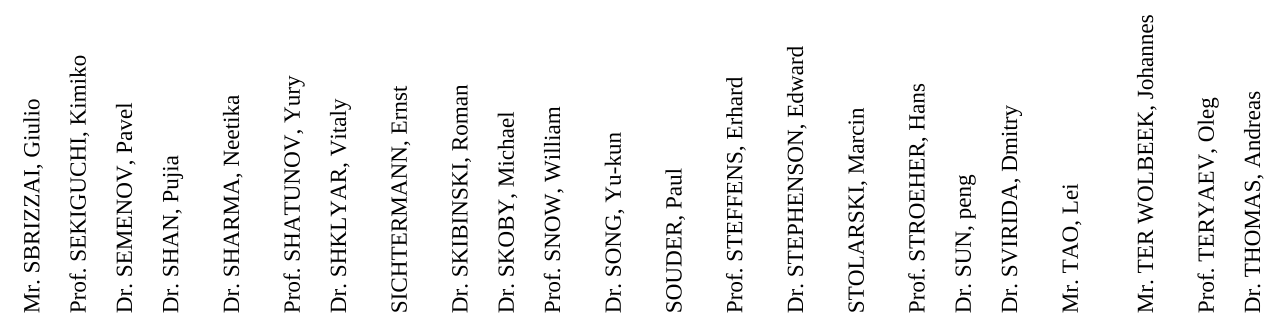




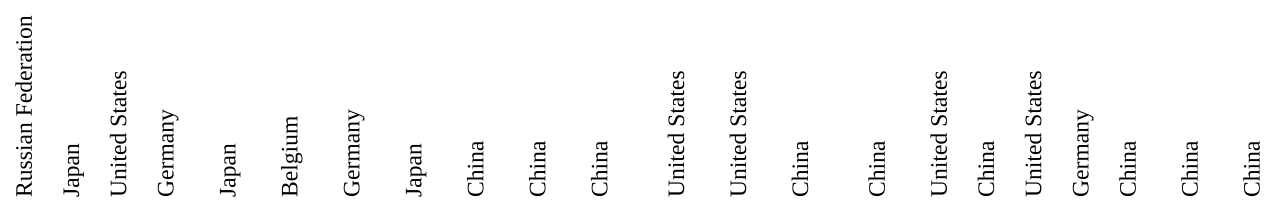

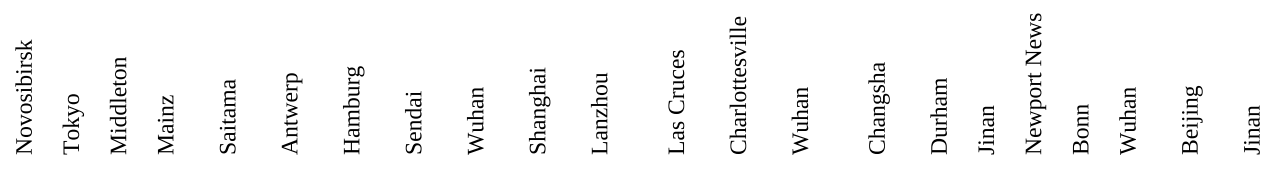

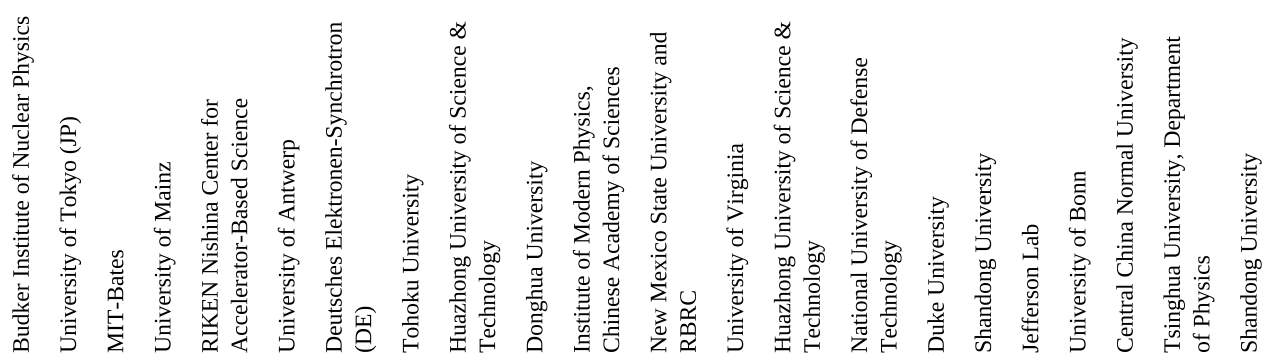

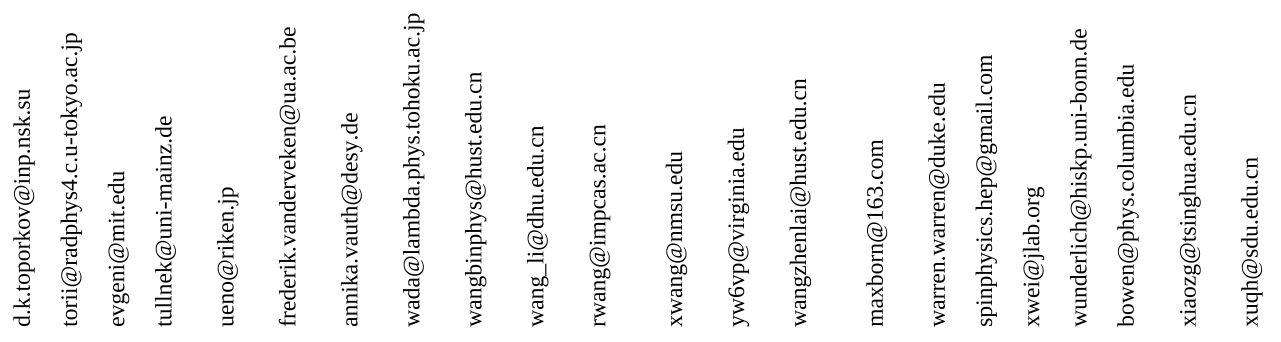

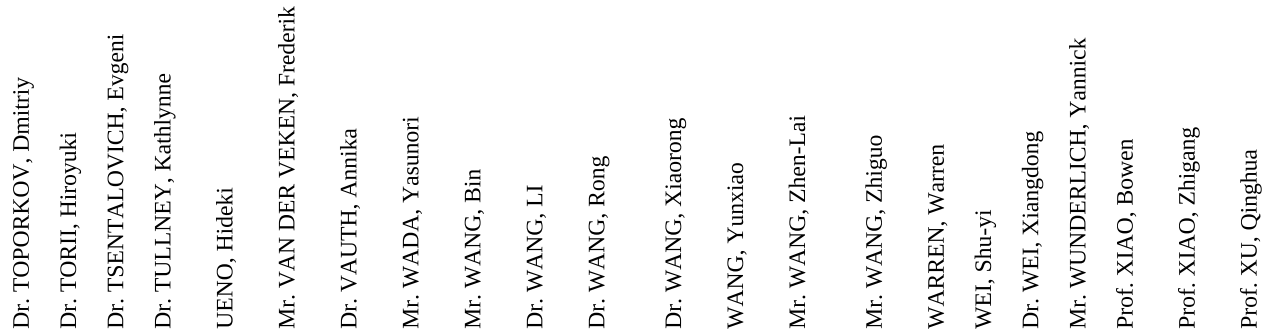




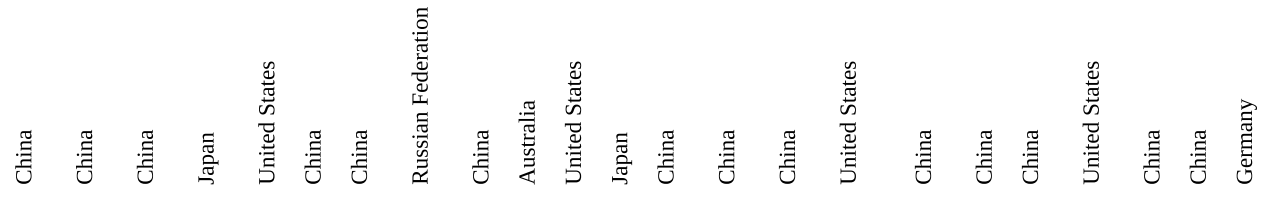

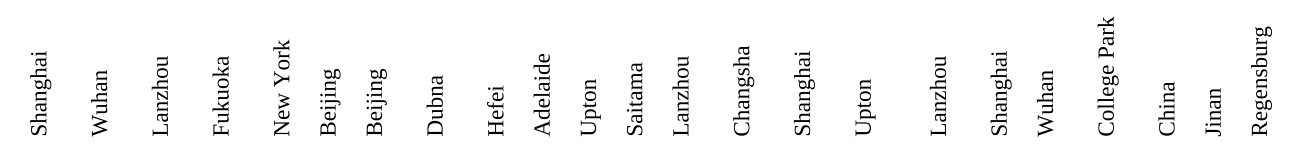

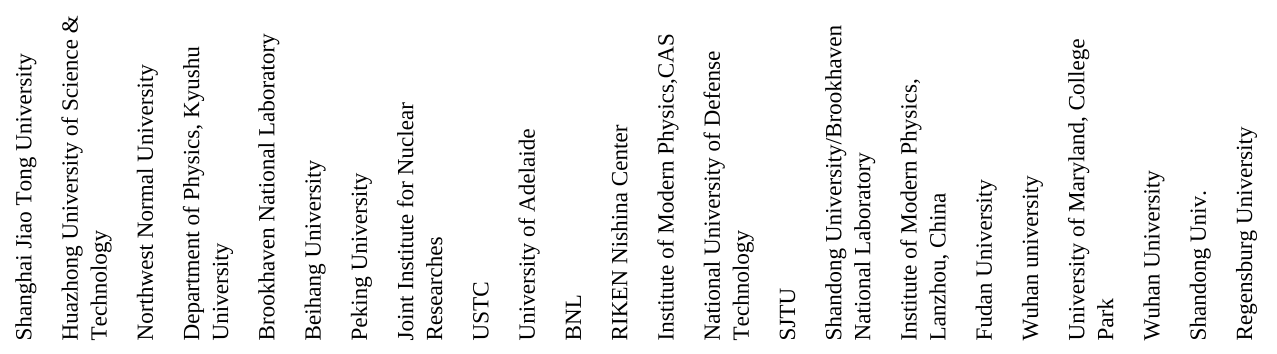

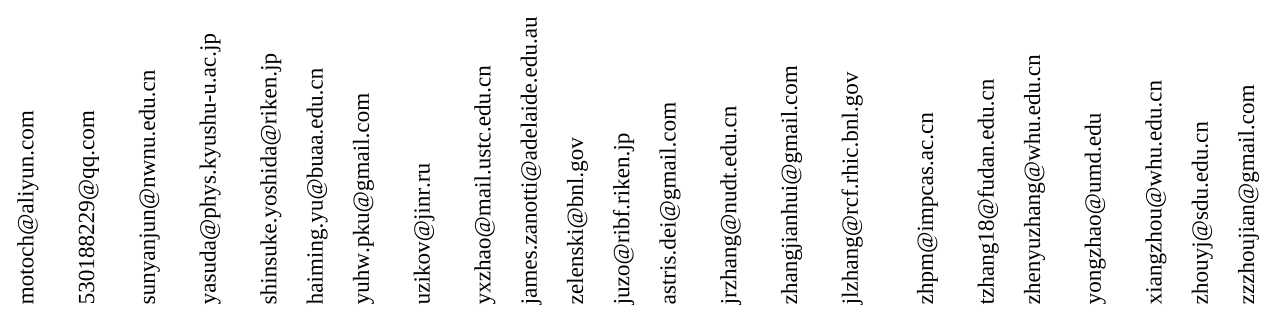

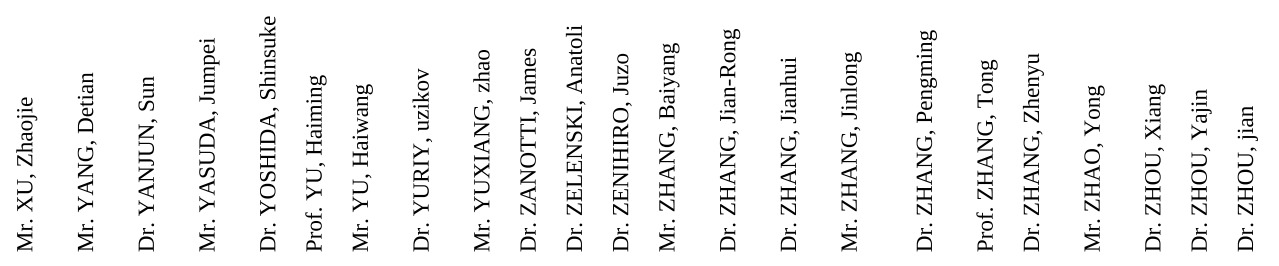




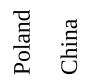

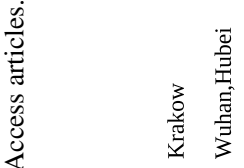

‡

0

to

包

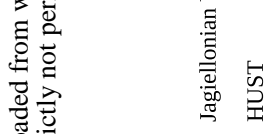
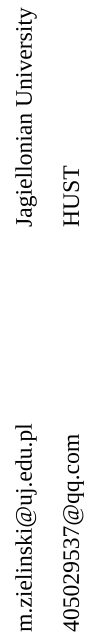

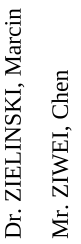

